\title{
Terceira Idade: documentação disponível na região metropolitana de Porto Alegre
}

\author{
Jussara PereiraS antos ${ }^{1}$ \\ Filipe X erxeneski da Silveira ${ }^{2}$
}

Este levantamento bibliográfico abrange material documental sobre terceira idade, envelhecimento velhice idoso aposentadoria, geriatria e gerontologia presente no acervo das Bibliotecas do Sistema de Bibliotecas da Universidade Federal do Rio Grande do Sul (UFRGS), Biblioteca Central da Pontifícia Universidade Católica do Rio Grande do Sul (PUCRS), Biblioteca Central da Universidade do Vale do Rio do Sinos (UNISINOS), Biblioteca do Instituto Multifuncional do Centro de Pesquisas e Documentação (CEDOPE) da UNISINOS, Biblioteca Central da Universidade Luterana do Brasil (ULBRA) e Biblioteca do Instituto Porto Alegre e Instituto Metodista de Ensino e Cultura (IPA/IMEC). A listagem abrange o material catalogado nas respectivas Bibliotecas até dezembro de 1998.

O levantamento foi realizado via Internet e/ou na própria Biblioteca depositária. A busca foi realizada a partir da classificação utilizada por cada Biblioteca, com base nos descritores apresentados acima. De posse do levantamento do acervo, foi procedida a digitação das referências bibliográficas em um banco de dados construido a partir do programa "CDS ISIS for Windows", versão de janeiro de 1998.

A listagem que segue é feita por ordem alfabética de autor. Cada referência é seguida pela indicação da localização da obra. Com relação a documentação pertencente à UFRGS, foram indicadas as Bibliotecas das Unidades onde se encontram, sem indicação da universidade.

\footnotetext{
${ }^{1}$ Professora da Faculdade de Biblioteconomia e Comunicação da UFRGS.

${ }^{2}$ Aluno do Curso de Biblioteconomia da UFRGS, bolsista do CNPq.
} 
1 ACORSI, Pedro Henrique. Marketing para a Promoção do Turismo de Terceira Idade na Região das Hortênsias. São Leopoldo, Unisinos, 1995. 45p. UNISINOS

2 ADAMS, Willian Carter. Foundations of Physical Education, Exercise, and Sport Sciences. Philad elphia, Lea Febiger, 1991. 296p. ESEF

3 ADRADOS, Isabel. Rorschach: teoria e prática do método na terceira idade. SãoPaulo, Casa do Psicólogo, 1988 180p. PSICO

4 ADRADOS, Isabel. Teoria ePrática do Teste de Rorschach. Rio de Janeiro, Fundação Getúlio Vargas, 1967. 461 p. PSICO

5 ALBUQUERQUE, Roseli Luarento de. Atividades Físicas e Recreativas como Busca de Realização Física e Emocional de Indivíduos na Terceira Idade. São Leopoldo, Unisinos, 1995. 94p. UNISINOS

6 ALEMAN, Luís Manuel Cornejo. PrevalênciadeMorbidadeClínica em Pacientes Maiores de 60anos. Porto Alegre, PUCRS, 1992 75f. PUCRS

7 AMERICAN COLLEGE OF SPORTS MEDICINE. Sport Sciences. Philadelphia, Lea Febiger, 1988 436p. ESEF

8 AMORIM, Paulo Roberto dos Santos. Educação Física na Terceira Idade. Brasília, SEED/MEC, 1989. p. 149-168. ESEF

9 ANDERSON, Linnea et al. Nutrición humana: princípios y aplicaciones. Barcelona, Bellaterra, 1977. 349p. ULBRA

10 ANDERSON, Margaret. Looking Ahead. New York, Basic Books, 1965. 252p. ULBRA

11 ANDRADE, José Hermógenes de. Saúde na Terceira Idade: saúde jovem, obra da natureza - saúde do idoso, obra de arte. Rio de Janeiro, Nova Era, 1997. 347p. CEDOPE.

12 ANDRADE, Luís Augusto Franco de. Alguns Aspectos Neurobiológicos do Envelhecimento. Ciênciae Cultura, São Paulo, v. 40, n. 7, p. 665-672,jul. 1988 UNISINOS

13 ANTONELLI, Paulo Ernesto. A Importância daDinâmica Corporal na Manutenção daSaúde e Melhoria da Qualidade de Vida, Voltada para a Faixa Etária entre 45e 72anos de Idade. Porto Alegre, PUCRS, 1996. 190p. ESEF 
14 ARIPE, Paulo. A Jovem no Ano Internacional do Idoso. Santa Maria, Paloti, 1982 24p. ULBRA

15 ASLAN, Ana. Os Mistérios de Envelhecer. Correio daUnesco, São Paulo, v. 10, n. 12 p. 89, dez. 1987. UNISINOS

16 ASSOCIAÇÃODOSDIPLOMADOSDA ESCOLA SUPERIORDEGUERRA. O Brasil Ante o Desafío dos Velhos. São Paulo, 1975. 107p. UNISINOS

17 AUCLAIR, Marcelle. Vida Jovem. Rio de Janeiro, Agir, 1971. 290 p. UNISINOS

18 AZEVEDO, Helena Alves D'. Atividades Aquáticas paraa TerceiraIdade. Porto Alegre, UFRGS, PROGRAD/PROPLAN, 1993 v. 2 p. 111. ESEF

19 BAGATINI, Vilson Fermino. Educação Física para Deficientes. Porto Alegre, Sagra, 1987. 359p. ESEF

20 BA GGIO, Marco Aurélio. Aspectos Psicológicos da Sexualidade do Idoso. Intercâmbio, Rio de Janeiro, v. 3, n. 7/ 9, p. 27-34, jan./ dez. 1990. EDU

21 BARBOSA, Vera Maria Barreto. Fisioterapia Geriátrica: prevenção e tratamento de problemas articulares na fase pré-geriátrica. Porto Alegre, Artes Médicas, 1983 46p. IPA/IMEC

22 BARRETT, James H. Gerontological Pysicology. Springfield, Charles C. Thomas, 1972. 165p. ULBRA

23 BARROS, Darcymieres do Rego. Ginástica Gerontológica: a reeducação psicomotora para pessoas idosas. Tubino, Manoel José Gomes. Homo Sportivus, ColeçãoEspecial deEducação Física e Desportos, Rio de Janeiro, v. 1, p. 41-44, 1984 1987. ESEF

24 BARROS, Myriam M. Lins de. Velhice ou TerceiraIdade? estudos antropológicos sobre identidade, memória e poética. Rio de Janeiro, FGV, 1998 235p. CEDOPE

25 BARROS, Myriam M. Lins de; ELIAS, Roseli. O Perfil dos Idosos no Município de Angra dos Reis. Cadernos do Ceas, Salvador, n. 141, p. 52-66, set./ out. 1992 UNISINOS

26 BARROS, Myriam Moraes Lins de; PRADO, Rosane Manhaes. Perspectivas Antropológicas daMulher. Rio de Janeiro, Zahar, 1981. 112p. EDU

27 BASTIAN, Ernestine Maurer. Internatos para Pessoas Idosas: uma avaliação. RevistaG aúchade Enfermagem, Porto Alegre, v. 7, n. 1, p. 123-131, jan. 1986. ENF 
28 BAUR, Robert (Org.) Ginástica, Jogos e Esportes para Idosos. Rio de Janeiro, Ao Livro Técnico, 1983، 280p. ESEF-UNISINIOS-IPA/IMEC

29 BEAUVOIR, Simone de. A Velhice. Rio de Janeiro, Nova Fronteira, 1990. 711 p. UNISINOS-IPA/IMEC-UFRGS

30 BECKER, Lauro da Silva; KNECHTEL, Maria do Rosário; MOKOWSKI, Tadeu C. Qual é mesmo a nossa Profissão? Blumenau, FURB, 1995. 56p. ULBRA

31 BENITEZ, Manoel Evangelista. Aspectos sociológicos de la adaptacion y convivência en la tercera edad. RevistaEspañoladeGerontologia, Madrid, v. 1, n. 5, p. 363-370, out. 1975. UNISINOS

32 BERENGI, Edith. A Boa Idade. Correio daUnesco, São Paulo, V. 15, n. 10, p. 25-27, out. 1987. UNISINOS

33 BERGER, Louise; MAILLOUX-POIRIER, Danielle. Pessoas Idosas: uma abordagem global processo de enfermagem por necessidades. Lisboa, Lusodidactica, 1995. 594p. ENF

34 BERGMANN, Claudine Consuelo. Desporto na Terceira Idade; uma proposta de voleibol adaptado. São Leopoldo, UNISINOS, 1996. 53 p. UNISINOS

35 BERMAN, Phillip L. A Coragem deEnvelhecer. São Paulo, Gente, 1989. 296p. UNISINOS-ULBRA-UFRGS

36 BERNHOEFT, Renato. Trabalhare Desfrutar. equilíbrio entre vida pessoal e profissional. São Paulo, Nobel, 1991. 126p. PUCRS

37 BERQUÓ, Elza Salvatori; LEITE, Valéria da Motta. Algumas Considerações sobre a Demografia da População Idosa no Brasil. Ciência eCultura. São Paulo, v. 40, n. 7, p. 679-688, jul./ 1988 UNISINOS

38 BINAI, Henri. O Eu e o Tempo: psicanálise do tempo e do env elhecimento. São Paulo, Casa do Psicólogo, 1991. 161 p. ULBRA

39 BITTENCOURT, Rosa Maria. A Problemáticae Sugestões para oLazer na Terceira Idade. São Leopoldo, UNISINOS, 1992 55p. CEDOPE

40 BIZE, P. R.; VALLIER, C. Uma Vida Nova: a terceira idade. Lisboa, Verbo, 1985. 235p. UNISINOS

41 BORGES, Nádia Sá. Aposentadoria, Trabalho eEducação: os imperativos do mundo da vida e do mundo do sistema. Porto Alegre, PUCRS, 1995. 116f. PUCRS 
42 BORN, Tomiko. A Dignidade na Terceira Idade. Tempo e Presença, São Paulo, v. 14, n. 14, p. 5-7, jul./ ago. 1992 EDU

43 BOSI, Ecléa. Memóriae Sociedade: lembranças de velhos. São Paulo, T. A. Qeiroz/Edusp, 1987. 402p. IPA/IMEC-UFRGS

44 BRA SIL, Ana Lia dos Santos. O Idoso naSociedade ea SociedadeIdosa: a importância do cuidade do corpo e com a mente e suas repercussões sociais. Porto Alegre, UFRGS/ Escola de Enfermagem, 1991. 23f. ENF

45 BRASIL. Direitos do Cidadão Idoso. São Paulo, Fundo Social de Solidariedade, 1992 32p. PUCRS

46 BRASIL. LEIS, DECRETOS, etc. Política Nacional doIdosoDeclaração Universal dos Direitos Humanos. Brasília, Sec. Nac. Dir. Hum., 1988 82p. ULBRA.

47 BRASIL. MINISTÉRIODA PREVIDÊNCIA EASSISTÊNCIA SOCIAL. Política Nacional do Id oso: Decreto n. 1.948de 3de julho de 1996. O Mundo da Saúde, São Paulo, v. 21, n. 4, p. 252-253 jul./ ago. 1997. ENF

48 BRINK, T. L. Psicoterapia G eriátrica. Rio de Janeiro, Finago, 1983 308 p. IPA/IMEC

49 BRITO, Carlos Roberto de. Reapropriação do Corpo do Idoso Através das A tividades Recreativas. Sprint Magazine, Rio de Janeiro, v. 11, n. 58, p. 6-9, jan./ fev. 1992 ESEF

50 BROCKLEHURST, J. C. GeriatriaFundamental. Barcelona, Toray, 1979. 289p. ULBRA

51 BÜCHELE, Maria da Graça S. Retalhos Históricos das Comunidades: grupo de idosos. Concórdia, Equiplan, 1995. 218p. UNISINOS

52 BULLA, Leonia Capaverde; CARLOS, Sergio Antonio; HEREDIA, Vania Beatriz Merlotti. As Condições de Vida dos Idosos do Rio Grande do Sul. In: A Pesquisa em ServiçoSocial enas Áreas Humano-Sociais. Porto Alegre, EDIPUCRS, 1998 p. 111-124 PSICO

53 BULLOUGH, Bonnie; BULLOUGH, Vern. Nursing in the Community. St. Louis, The C. V. Mosby, 1990. 712p. ENF

54 BURNSIDE, Irene Mortenson. Enfermagem e os Idosos. São Paulo, Organização Andrei Editora, 1979. 547p. UFRGS-CEDOPE

55 BUTLER, Robert N.; LEWIS, Myrna I. Sexoe Amor na TerceiraIdade. São Paulo, Summus, 1985. 135p. PUCRS-EDU 
56 CAMPEDELLI, Maria Coeli. Consulta de Enfermagem em Geriatria: tópicos a serem abordados na entrevista - parte I. Revista daEscoladeEnfermagem da USP, São Paulo, v. 25, n. 3, p. 319-33, dez. 1991. ENF

57 CAMPEDELLI, Maria Coeli. Consulta de Enfermagem em Geriatria: tópicos a serem abordados no exame físico - parte II. Revista da Escola de Enfermagem da USP, São Paulo, v. 26, n. 1, p.33-42, mar. 1992 ENF

58 CANOAS, Cilene Swain. A Condição Humana do Velho. São Paulo, Cortez, 1985.79p. UFRGS-UNISINOS

59 CANTO, Silvia Helena Oliveira do. Aposentadoria: um fim que exige um recomeço. São Leopoldo, Unisinos, 1983 54p. CEDOPE

60 CARNEGIE, Dorothy. A Maturidade: segredos da eterna juventude. São Paulo, Companhia Editora Nacional, 1980. 213p. UNISINOS

61 CARNEIRO, Maria Verônica. Sexualidade: o fantasma da terceira idade. Intercâmbio, Rio de Janeiro, v. 4, n. 10, p. 5-14, jan./abr. 1991. EDU

62 CARNEY, Joyce M. A Educação de Pré-Aposentados. Rio de Janeiro, Rocco, 1981. 14p. PUCRS

63 CARROLL, Mary; BRUE, L. Jane. Enfermagem para os Idosos. São Paulo, Organização Andrei Editora, 1991. 195p. ENF

64 CASTRO, Carla Renata Nogueira de; RODRIGUES, Rosalina Aparecida Partezani. O Idoso e a A posentadoria. Revista da Escola de Enfermagem da USP, São Paulo, v. 26, n. 3, p. 275-88, dez. 1992 ENF

65 CENTRO LATINOAMERICANO DE DEMOGRAFIA. Argentina: Situación y necesidades de la tercera edad: algunas ciudades seleccionadas. Santiago de Chile, CELADE, 1989. 195p. DIR

66 CHACIN, Migdy N. Expectativas de los facilitadores y logros universitários en la tercera edad. Planiuc, Valência, v. 8/ 10, n. 15/ 17, p. 139 -147, Ene./ Dic. 1991. EDU

67 CHATKIN, Jose Miguel; MENNA BARRETO, Sérgio Saldanha. Asma: liberdade para respirar: guia de orientação para pais e pacientes. Porto Alegre, Artes Médicas, 1996 105p. MED

68 CHA VEZ SAMPERIO, Jorge. La discapacidad fisico-funcional en el pensionado. Ciudad de Mexico, DDF, 1994 p. 72-80. ESEF.

69 COLLANGE, Christiane. Eu sua Filha. Rio de Janeiro, Rocco, 1992160 p. UNISINOS 
70 COMBAS, Christian. O Elogio da Idade em um Mundo Bronzeado. Rio de Janeiro, Nova Fronteira, 1990. 156p. IPA/IMEC

71 CONFORT, Alex. A BoaIdade. São Paulo, Difel, 1979. 232p. UNISINOS

72 CONGRESSO BRASILEIRO DE SAÚdE COLETIVA, 2, 1989, São Paulo; CONGRESSO PAULISTA DE SAÚDE, PÚBLICA, 3, 1989, São Paulo. Programa doSistema Único deSaúde, São Paulo, Associação Brasileira de Pós-Graduação em Saúde Coletiva, 1989. 118p. ENF

73 CORRÊA, Antônio Carlos de Oliv eira. Envelhecimento, Depressão e Doença de Alzheimer. Belo Horizonte, Health, 1996 227p. PSICO

74 COSTA, Laís Martins de Menezes. O Exercício na Prevenção de Osteoporose naMulherPós-Menopausa Porto Alegre, UFRGS/ESEF, 1996. 40f. ESEF

75 COSTA, Veraluza Martins; CAVALCANTI, Katia Brandão. A Reeducação Psicomotora na Terceira Idade: um estudo de caso com idoso marginalizado. Intercâmbio, Rio de Janeiro, v. 4, n. 11/ 12 p. 23-28, maio/dez. 1991. EDU

76 CRUZ, Maria Zilda da. O Id oso: delimitações da velhice. Leopoldianum, Santos, v. 17, n. 49, p. 5-21, abr./ 1991. EDU

77 CUIDADOS com Medicamentos. Porto Alegre, Ed. da Universidade/ UFRGS, 1996. 173p. PUCRS

78 CULTURA en la tercera edad. São Paulo, Ministério da Cultura, 1983 117p. UNISINOS

79 CUSATO, Leonardo. PrevidênciaSocial: normas e cálculos de benefícios. Porto Alegre, Sagra, 1988 108p. ULBRA

80 DANTAS, Estélio Henrique Martins. A Corrida de Longa Duração na Terceira Idad e. Coleção Especial de Educação Física e Desportos, Rio de Janeiro, v. 2 , n. 1, p. 73-92, 1984 1987. ESEF

81 DEBERT, Guita Grin. Desbravando Fronteiras e Redefinindo Padrões. Tempo e Presença, São Paulo, v. 14, n. 264, p. 1325, jul./ ago. 1992 EDU

82 DIRIA, Alberto, (Coord.) The OlympicBook of Sports Medicine. Oxford, Blackw ell, 1988. 692p. ESEF

83 DIZ, Digo. A última Idade: a arte de envelhecer. Braga, Editorial AO, 1981. 124p. UNISINOS 
84 EDITORIAL de La Civilta Católica. A Idade Inútil: o problema dos anciões na sociedade industrial. Cultura e Fé, São Paulo, v. 10, n. 18, p. 3651, jul./ set. 1982 UNISINOS

85 EDUCAÇÃ O e Terceira Idade. Correio daUnesco, São Paulo, v. 10, n. 12 p. 28-31, dez. 1982 UNISINOS

86 EDWARDH, Joey; MORENO A., Cecilia (Ilust.) Latercera edad: victima de mitos sociales o protagonistas en la transformación de la sociedad, un nuevo frente de trabajo para la educacion popular. Santiago, CEAAL, 1987. 110p. EDU

87 EISELE, Loriane. A Aposentadoria na Terceira Idade. São Leopoldo, Unisinos, 1994 30p. CEDOPE

88 ELKOWITZ, Edw ard B. Primeiros Socorros ao Paciente Geriátrico. São Paulo. Andrei, 1985. 252p. IPA/IMEC

89 EPSTEIN, Charlotte. InteraçãoEfetiva naEnfermagem. São Paulo, EPU, EDUSP, 1977. 173p. ENF

90 ESPIRITO, Poli Marcelino. O Exercício Físico naPrevenção de Distúrbios Geriátricos. Porto Alegre, Artes Médicas, 1971. 12p. IPA/IMEC

91 EX-ATLETAS Precisam Manter o Ritmo da Juventude. Sprint Magazine, Rio de Janeiro, v. 9, n. 51, p. 23, nov./dez. 1990. ESEF

92 FACHIN, Odília. Aspectos Sociológicos daVelhice. São Paulo, Gente, 1975. 224p. UNISINOS

93 FARACO, Daniel. Previdência Social - bomba de tempo?CulturaeFé, São Paulo, v. 4, n. 13, p. 61-70, abr./jun. 1981. UNISINOS

94 FARACO, Daniel. Previdência Social: algo semelhante ao petróleo. Cultura eFé, São Paulo, v. 2 n. 7, p. 92-94, out./ dez. 1979. UNISINOS

95 FARIA, Carlos Coelho de. A Vida não tem Idade: uma experiência a serviço da Gerontologia Social. São Paulo, Depto. de Geriatria Dom Pedro II, 1983. 288p. UNISINOS

96 FAVERO, Cleci Eulália. História Oral / História de Vida: das dificuldades de uma pesquisadora na coleta de depoimentos de mulheres velhas. Estudos Leopoldenses, São Leopoldo, v. 32 n. 146, p. 101-108, mar./abr. 1996. EDU

97 FERNANDES, Flávio da Silva. As Pessoas Idosas daLegislação Brasileira (Direitoe G erontologia). São Paulo, LTR, 1997. 167p. ULBRA 
98 FERRARI, Maria Auxiliadora Cursino. Idade Avançada: nova preocupação da saúde pública. São Paulo, 1981. 117p. IPA/IMEC

99 FERRARI, Maria Auxiliadora Cursino. O Terapeuta Ocupacional em Geriatria e Gerontologia. Anais Brasileiros de Geriatria e Gerontologia, SãoPaulo, v. 3, n. 1, p. 2-6, fev. 1981. ENF

100 FERREIRA, Berta Weil. Considerações sobre a Velhice. Veritas, Porto Alegre, v. 24, n. 93, p. 87-95, mar. 1979. EDU

101 FERREIRA, Odson Costa. Idoso no Brasil. Sprint Magazine, Rio de Janeiro, v. 15, n. 85, p. 18-21, jul./ ago. 1996 ESEF

102 FERRIGNO, José Carlos. A Sexualidade dos mais Velhos. Intercâmbio, Rio de Janeiro, v. 3, n. 7/ 9, p. 35-45, jan./ dez. 1990. EDU

103 FILIZZOLA, Mário. ComoEmplacar 100anos. Rio de Janeiro, O Cruzeiro, 1966. 220p. CEDOPE

104 FITZGERALD, Patrick L. O Exercício para a Terceira Idade. Sprint Magazine, Rio de Janeiro, v. 12 n. 65, p. 9-13, mar./ abr. 1993 ESEF

105 FLORES, Maria Elias de Souza. A Dignidade de Envelhecer. São Leopoldo, Unisinos, 1995. 98p. CFDOPE

106 FLOSI, Attilio Zelante. Tratamento do Climatério Feminino. São Paulo, Medisa, 1975. 64p. PUCRS

107 FOLBERG, Maria Nestrovsky; SOUZA, Tatiana Magalhães. Velhos Falam e são Ouvidos: flashes de uma pesquisa com a terceira idade. Educação, Porto Alegre, v. 17, n. 26, p. 81-87, 1994. EDU

108 FORCIEA, Mary Ann; LAVIZZO-MOUREY, Risa. Segredos em G eriatria. Porto Alegre, Artes Médicas, 1998 328p. MED

109 FORJAZ, Marina V. Enfermagem no Lar: noções práticas. São Paulo, Ed. Nacional, [1962?] 187p. ENF

110 FORMIGA, Nara Becker. O Sentimento de Rejeição na TerceiraIdade. São Leopoldo, Unisinos, 1989. 83p. UNISINOS

111 FRADINHO, Manuel. A Cultura Física em Geriatria Lisboa, Gazeta Médica Portuguesa, 1959. 4p. IPA/IMEC

112 FRAIMAN, Ana Perwin. Sexo e Afeto na TerceiraIdade; aquilo que você quer saber e não teve com quem conversar. São Paulo, Gente, 1994313 p.CEDOPE-PSICO-IPA/IMEC 
113 FRANCA, Lúcia Helena de Freitas Pinho. O Resgate do Tempo. Boletim Técnico doSenac, Rio de Janeiro, v. 15, n. 2 p. 97-111, maio/ago. 1989. EDU

114 FRANCA, Lúcia Helena de Freitas Pinho. Perspectivas Existenciais em um Grupo de Idosos. Intercâmbio, Rio de Janeiro, v. 3, n. 7/ 9, p. 13-25, jan./dez. 1990. EDU

115 FREIRE, Gilberto. O Idoso Válido como Descoberta de nossa Época. Ciência e Trópico, Recife, v. 5, n. 1, p. 65-78, jan./jun. 1977. UNISINOS

116 FREITAS, Jussara da Silva. Ações de Enfermagem esuaImportância segundo o Enfermeiro e o Paciente G eriátrico. São Leopoldo, Unisinos, 1992. 65p. UNISINOS

117 FRENTZEL, Carla Leandro Demarchi. A Cirurgiae a Traumatologia Bucomaxilofacial frente aos Pacientes Idosos. Porto Alegre, Artes Médicas, 1997. 120f. PUCRS

118 FRONTERA, Walter; BECERRO, J.; GOMEZ, R.. Salud y la actividad física en las personas. Barcelona, Paudotribo, 1987. [s.p]. ULBRA

119 GAIARSA, José Angelo. ComoEnfrentar a Velhice. São Paulo, Ícone, 1993 87p. PUCRS-CEDOPE-IPA/IMEC-UFRGS

120 GEIST, Harold. Psicologia y psicopatologia del envejecimiento. Buenos Aires, Paidos, 1977. 149p. UNISINOS

121 GIL, Lourdes da Silva. Depoimentos de Pessoas Idosas sobre sua Atividade Cognitiva: implicações educacionais. Porto Alegre, PUCRS, 1991. 286f. PUCRS

122 GOLDIM, José Roberto. Bioética e Envelhecimento. Gerontologia, v. 5, n. 2 p. 66-71, 1997. MED

123 GOMES, Fredefico Alberto de Azevedo. Manual de Geriatria e Gerontologia Rio de Janeiro, EBM, 1985. 301p. IPA/IMEC

124 GRA Y, Madeline. The Changing Years. New York, Signet Book, 1970. 237p. ULBRA

125 GRAY, Roberto M.; MOBERG, David O. Church and the Older Person. Michigan, Willian B. Federman, 1977. 227p. ULBRA

126 GUILLY, Paul. Laedad critica Buenos Aires, EUDEBA, 1959. 64p. PUCRS

127 GUZINSKI, Maria A parecida Magnante. PolíticaSocial para oIdoso Carente no GovernoBorges de Medeiros: 1898 1928 Porto Alegre, PUCRS, 1995. 207 f. PUCRS 
128 HADDAD, Eneida Gonçalves de Macedo. A Ideologia da Velhice. São Paulo. Cortez, 1986 134p. UNISINOS

129 HADDAD, Eneida Gonçalves de Maced o. O Direitoà Velhice: os aposentados e a previdência social. São Paulo, Cortez, 1993 115 p. PUCRSUNISINOS-IPA/IMEC

130 HADDAD, Eneida Gonçalves de Macedo. Rompendo o Cerco. Tempo e Presença. São Paulo, v. 14, n. 264, p. 22-24, jul./ ago. 1992 EDU

131 HADDAD, Eneida Gonçalv es de Macedo. A Velhice de Velhos Trabalhadores. Cadernos CERU. São Paulo, v. 2 n. 6, p. 76-88, 1995. UNISINOS

132 HAESBAERT, Karin Vera. Terceira Idade. São Leopoldo, Unisinos, 1993. 278p. CEDOPE

133 HAMADA, Gerson Shigueaki; MORIGUCHI, Yukio; NASCIMENTO, Evaldo Carlomagno. Características das Doenças do Idoso. Revista de Medicina daPUCRS. Porto Alegre, v. 2 n. 3, p. 170 174, 1990. PUCRS

134 HANSEN, Patrícia. Tasks of Residential Workers: a study of direct care practitioners. Aldershot, Avebury, 1988, 170p. PSICO

135 HAYFLICK, Leonard. Como e por que envelhecemos. Rio de Janeiro, Campus, 1996, 366p. PSICO

136 HENS, Vanise. O Perfil do Idoso num Hospital Psiquiátrico. São Leopoldo, Unisinos, 1995, 181p. UNISINOS

137 HERMÓGENES, José. SaúdenaTerceiraIdade. Rio de Janeiro, Record, 1996, 347p. IPA / IMEC

138 HILDEBRANDT, Reiner; COSTA, Vera Luzia Lins. A Pessoa Idosa e Fenômeno Esportivo: uma abordagem didática. São Cristóvão, Aracaju, v. 2 n. 3, p. 118 121, jan. 1990. ESEF

139 HOOD, Grail Harkeness; DINCHER, Judith R. Fundamentos e PráticadeEnfermagem; atendimento completo ao paciente. Porto Alegre, Artes Médicas, 1995. 769p. UNISINOS

140 HOOKE, Ann P. Active Older Adults in the YMCA: a resources manual. Champaign, YMCA of the USA, Human Kinetics, $1992301 \mathrm{p}$. ESEF

141 HOWELL, Sandra C. Designing for Aging: patterns of use. London, Mit Press, 1987. 329p. ARQ 
142 HULME, Willian E. Vintage Years: growing older with meaning and hope. Philad elphia, Westminister, 1986. 120p. ULBRA

143 IDOSOS uma questão nacional. Planejamento e Desenvolvimento, Rio de Janeiro, v. 4, n. 11, p. 13-17, dez. 1976. UNISINOS

144 JANI-LE BRIS, Hannelore. Prise en Charge Familiale des Dependants Ages ans les Pays des Communautes Europeennes. Dublin, Loughlinstown, 1993.186p. DIR

145 JOHNSON, Anna Alzira Conter. A Mulher Idosa e a Expectativa de Futuro. São Leopoldo, Unisinos, 1992 68p. UNISINOS

146 JUNQUE, Carme; JURADO, Maria Angeles. Envejecimiento y demencias. Barcelona, Martinez Roca, 1994 207p. ULBRA

147 KABWASA, Nsang O'khan. O Eterno Retorno. Correio da Unesco, São Paulo, v. 10, n. 12, p. 14 15, dez. 1987. UNISINOS

148 KANE, Robert L. O Essencial em Clínica Geriátrica. São Paulo, Andrei, 1985. 346p. IPA/IMEC

149 KASTENBAUM, Robert. Velhice: anos de plenitude. São Paulo, Harpor Hao, 1979. 128p. IPA/IMEC

150 KATZ, David, et al. Psicologia das Idades: do nascimento a morte. São Paulo, Manole, 1988 114p. EDU

151 KEITH, Arthur. A BoaIdade. São Paulo, Círculo do Livro, 1997. 232p. ULBRA

152 KLLINEN, Mauri; MARU, Alen. Lesões Esportivas na Terceira Idade. Sprint Magazine, Rio de Janeiro, v. 15, n. 84, p. 8-18, maio/jun. 1996. ESEF

153 KOKUBUN, Eduardo. O Consumo Máximo de Oxigênio ao Longo do Ciclo Vital. In: Reunião Anual da Sociedade Brasileira para o Desenvolvimento da Educação Física, 2, 1991, Rio Claro. [Anais...]. Rio Claro, SBDEF, 1991. p. 62-67 ESEF

154 KOLLING, Aloysio. O Homem da Terceira Idade também deve praticar Educação Física. Revista de Estudos, Novo Hamburgo, v. 5, n. 1, p. 35 38, jun. 1982 EDU-ESEF

155 KOZLOV, Victor. Receitas para Viver 100anos. Correio da Unesco, São Paulo, v. 10, n. 12, p. 10-13, dez. 1987. UNISINOS 
156 KROEFF, Locimara Ramos, et al. Salão de Iniciação Científica. Resgate de uma Interação entre os Objetivos da Universidade para a Terceira Idade (UNITI) e as Expectativas de seus Alunos com relação a mesma. In: SALÃO DE INICIAÇÃ O CIENTÍFICA, 5, 1993, Porto Alegre. [Resumos]. Porto Alegre, UFRGS, 1993. p. 262. PSICO

157 KUNZ, Sigrid. Implantação de um Programa de Recreação e Lazer para os Idosos da SPAAN. Porto Alegre, UFRGS, 1982 63f. ESEF

158 LA PAROLE Est Aux Aidants: entretiens avec des aidants familiaux qui prennent en charge des personnes agees dependantes dans la communaute europpenne. Dublin, Loughlistown, 1994 130p. DIR

159 LA VEJEZ como problema social. Crônica de la OMS, Lisboa, v. 16, n. 28, p. 534542, 1974 UNISINOS

160 LAGES, Tatiana Ferreira. Terceira Idade: perfil dos freqüentadores do grupo de idosos Rosas de Maio/ FESC na cidade de Porto Alegre-RS. São Leopoldo, Unisinos, 1996. 102p. UNISINOS

161 LANTZ, Débora. Depressão na Terceira idade: uma caracterização teórico-prática. São Leopold o, Unisinos, 1993، 83p. UNISINOS

162 LEA, Magdalena. Quem tem MedodeEnvelhecer?São Paulo, Harbra, 1978. 282p. ULBRA

163 LEAL, Maria das Graças Sobreira. O Envelhecer na Instituição Religiosa Feminina. São Paulo, PUCSP, 1994 252p. PSICO

164 LEÃ O, Cláudia Sheibe; HEKMAN, Paulo Rogério Wasserstein. Avaliação Ecocardiográfica da Dimensão do Átrio Esquerdo de uma Amostra de Mulheres com Idade Superior a 65anos e Portad oras de Hipertensão Essencial. Revistade Medicina da PUCRS, Porto Alegre, v. 4, n. Esp., p. 15, 1994 PUCRS

165 LECER, J. M. Psicopatologia doEnvelhecimento: assistência aos idosos. Petrópolis, Vozes, 1996. 296p. IPA/IMEC

166 LEFEBRE, Helene. A A tualização da Pessoa Idosa pela Educação Continuada. Revista Portuguesa de Pedagogia, Coimbra, v. 23, p. 75-85, 1989. EDU

167 LEGER, J. M., et al. Psicopatologias do Envelhecimento: assistência às pessoas idosas. Petrópolis, Vozes, 1994 296p. CEDOPE

168 LEITE, Celso Barroso. O Século da Aposentadoria. São Paulo, LTR, 1993. 152p. ULBRA 
169 LEITE, Paulo Fernando. Aptidão Física: esporte, saúde, prevenção e reabilitação de doenças. São Paulo, Robe, 1990. 280p. ULBRA

170 LEITE, Paulo Fernando. Exercício, Envelhecimento e Promoção da Saúde. Belo Horizonte, Health, 1996 125p. UNISINOS

171 LEME, Luiz Eugênio Garcez. O Envelhecimento. São Paulo, Contexto, 1998. 70p. CEDOPE

172 LEMES, Iara Maria Abreu. O Idoso: trabalho, aposentadoria, traba1ho. São Leopoldo, Unisinos, 1994 60p. UNISINOS

173 LEMLE, Alfred. Pneumonias na Velhice. St. Louis, Concórdia, 1978. 126p. ULBRA

174 LERRIGO, Charles H. The Better Half of your Life. New York, JDay, 1951. 270p. ULBRA.

175 LIMA, Ângela Maria Machado de, et al. Programação em SaúdeHoje. São Paulo, ED. HUCITEC, 1990. 226p. ENF

176 LIMA, David de. A Atividade Física e sua Relação com a Auto-Imagem e Auto-estima na Terceira Idade. Porto Alegre, UFRGS, 1989. 100p. EDU-ESEF

177 LIMON MENDIZABAL, Maria Rosário. Reflexiones sobre la educación en la tercera edad. Revistade Educacion, Mad rid, n. 291, p. 225-251, Ene./ Abr. 1990. EDU

178 LORDA PAZ, C. Raul. Educação Física e Recreação para a Terceira Idade. Porto Alegre, Sagra, 1990. 135p. ESEF

179 LORDA, C. Raul; SANCHEZ, Cármen Délia. Recreação na Terceira Idade. Porto Alegre, Sagra, 1996 135p. ULBRA

180 MACHADO, Marta Berne. Úlcera Péptica Gástrica no Idoso: aspectos clínicos, complicações e características endoscópicas. Porto Alegre, PUCRS, 1995. 107 p. PUC

181 MAGALHÃES, Dirceu Nogueira. Envelhecimento e Resistência Cultural. Intercâmbio, Rio de Janeiro, v. 3, n. 7/ 9, p. 47-54, jan./ dez. 1990. EDU

182 MAGUIRE, Gail Hills. Care of the Ederly: a health team approach. Boston, Little Brown, 1985. 321 p. ULBRA

183 MAHAN, K. Kathleen. Krause. Alimentos, Nutrição eDietoterapia. São Paulo, Roca, 1995. 957p. IPA/IMEC 
184 MAKHOUL, Georges A. O Enigma do Amanhã. São Paulo, Hemus, [1990?]. 62p. PUCRS

185 MANKOWITZ, Ann. Menopausa: tempo de renascimento. São Paulo, Paulinas, 1987. 149p. PUC

186 MARDEGAN JR, Elyseu. A IdadedoLobo. São Paulo, Mercuryo, 1993. 102p. ENF-PSICO

187 MARTINEZ, Wladimir Novaes. Aposentadoria Especial. São Paulo, LTR, 1988 279p. ULBRA

188 MARTINEZ, Wladimir Novaes. Direito dos Idosos. São Paulo, LTR, 1997. 150p. ULBRA

189 MARTINS, Edilson. Os Velhos na Comunidade Indígena. Senecta, Rio de Janeiro, v. 3, n. 2 p. 4 10, 1980. UNISINOS

190 MARTINS, Eduardo Teles Castro. Exercício Físico na Terceira Idade. Revista Portuguesa de Medicina Desportiva, Lisboa, v. 8, n. 49, p. 288-294, nov/dez. 1990. ESEF

191 MAURER, Lucinda Appel. Os Sentimentos da Pessoa Idosa: implicações para uma educação para a saúde. Porto Alegre, UFRGS, 1990. 332p. EDU

192 MAZO, Giovana Zarpellon. A tividade Física: atitude dos idosos frente ao envelhecimento. Kinesis, Santa Maria, n. 7, p. 77-99, jun. 1991. ESEF

193 MEDEIROS, Esmeralda Aparecida Colombo. A Vivência e a Representação daImagem Corporal em Mulheres deIdade Avançada. São Paulo, USP, 1988. 182 p. EDU

194 MEDEIROS, Esmeralda A parecida Colombo. Mulherna TerceiraIdade: uma tentativa de levantamento de determinantes de solidão. Campinas, Pontifícia Universidade Católica de Campinas, 1983 277p. EDU

195 MEDEIROS, Esmeralda Aparecida Colombo. Psicoprofilaxia no Processo do Desenvolvimento: uma ação integrada. Estudos dePsicologia Campinas, v. 9, n. 1, p. 66-91, jan./abr. 1992 EDU

196 MEDEIROS, Osiris A. Borges de. Aposentadoria ao Alcance de Todos. Rio de Janeiro, Forense, 1968 215p. PUCRS

197 MEDIONDO, Marisa Silvana Zazzetta de. Os Costumes na VidaCotidiana dos Idosos: possibilidades e limites. Porto Alegre, 1996. 239f. PUCRS 
198 MEIRELLES, Morgana. Atividade Física naTerceiraIdade: uma abordagem sistêmica. Rio de Janeiro, Sprint, 1997. 109p. ULBRA-IPA/IMEC

199 MENOPAUSA e Pós Menopausa: guia clínico. [s.l, s-n, 1974?]. 63p. PUCRS

200 MEUSEL, Heinz. Dokumentationsstudie: Sport im Alter. Schriftenreihe, Karl Hofmann, 1980. 398p. ESEF

201 MODIFICAÇÕES do Exercício para a Terceira Idade. Sprint Magazine, Rio de Janeiro, v. 11, n. 62 p. 17- 18, set./ out. 1992 ESEF

202 MORAGAS, Ricardo. G erontologiaSocial; envelhecimento e qualidade de vida. São Paulo, Paulinas, 1997. 283p. CEDOPE

203 MORAIS, Maria da Graça. A Substituição das Gerações em Portugal: análise regional (1930-1975). Análise Social, Lisboa, v. 19, n. 75, p. 79-99, 1983. UNISINOS

204 MORGAN, John C. Becoming Old: an introduction to social gerontology. New York, Springer, 1979. 94p. ULBRA

205 MORIGUCHI, Yukio; MORIGUCHI, Emílio Hideyuki. BiologiaG eriátrica Ilustrada. São Paulo, Fundação BYK, 1988 239p. ULBRA

206 MOTTA, Flávia de Mattos. Velhaéa Vovozinha: identidade feminina na velhice. Porto Alegre, UFRGS, 1990. 183f. BSCSH-PSICO

207 MOURINO MOSQUERA, Juan José. Pessoa Idosa: problemas ou esperança? Veritas, Porto Alegre, v. 38, n. 149, p. 113 131, mar. 1993 EDU

208 NACIONESUNIDAS. CENTRODEDESENVOLVIMENTOSOCIALE ASSUNTOSHUMANITÁRIOS. Manual de organizaciones que desarrollan actividades en la esfera del envejecimiento. New York, 1989. 142p. DIR

209 NAHEMOV, Lucille; POUSADA, Lidia; ROTHENBERG, Mace. Diagnóstico Geriátrico: um estudo de casos. São Paulo, Organização Andrei Editora, 1987. 398p. ENF

210 NASARETH, J. Manoel. Dinâmica da População Portuguesa. Análise Social, Lisboa, v. 14, n. 56, p. 729-800, 1978 UNISINOS

211 NASCIMENTO, Daniela Meirelles do. Qualidade de Vidae Exercício Físico na TerceiraIdade. Porto Alegre, UFRGS/ESEF, 1996 20f. ESEF.

212 NASCIMENTO, Evaldo Carlomagno do. Tópicos em G eriatria Porto Alegre, PUC, 1984 158p. ULBRA 
213 NASH, Bernard E. Troca de Informações em Escala Mundial sobre o Envelhecimento. Centro de Documentação sobre a Terceira Idade. São Paulo, n. 11, p. 1-9, dez. 1976. UNISINOS

214 NEHER, Bruno. Cartilha da Terceira Idade. Porto Alegre, AL/RGS, 1988 47p. ULBRA

215 NERI, Anita Liberalesso (Org.) PsicologiadoEnvelhecimento. Campinas, Papirus, 1995. 276p. UNISINOS

216 NERI, Anita Liberalesso (Org). Qualidade de Vidae Idade Madura Campinas, Papirus, 285p. UNISINOS

217 NERI, Anita Liberalesso. Atitudes em Relação à Velhice: uma revisão de literatura: 1. parte - estudos interculturais. Estudos dePsicologia, Campinas, v. 6, n. 1, p. 75-93, 1989. EDU

218 NERI, Anita Liberalesso. Atitudes em Relação à Velhice: uma revisão de literatura: 2 parte - estudos investigando variáv eis individuais de natureza psicológica e sociológica. Estudos de Psicologia, Campinas, v. 6, n. 2 p. 41-61, 1989. EDU

219 NERI, Anita Liberalesso. Atitudes em Relação à Velhice: uma revisão de literatura: 3. parte - conclusões e sugestões para pesquisa no Brasil. Estudos de Psicologia, Campinas, v. 7, n. 1, p. 107-111, jan./mar. 1990. EDU

220 NERI, Anita Liberalesso. Chinelo Velho para Pé Cansado. Tempo e Presença, São Paulo, v. 14, n. 264, p. 8-9, jul./ ago. 1992 EDU

221 NERI, Anita Liberalesso. Envelhecer num País de Jovens: significados de velho e velhice segundo brasileiros não idosos. Campinas, Universidade de Campinas, 1991. 155p. UNISINOS

222 NERI, Anita Liberalesso. O Rótulo Social na Quarta Idade e as Implicações de seu Uso para o Estudo da Velhice no Brasil. Estudos de Psicologia, Campinas, v. 8, n. 1, p. 148-150, jan./jul. 1991EDU

223 NERI, Anita Liberalesso; NOGUEIRA, Eliete Jussara. Como a Velhice é A presentada às Crianças, em Textos de Literatura Infantil Brasileira. PróPosições, Campinas, v. 5, n. 1, p. 45-60, mar. 1994. EDU

224 NETO, Antônio Jordão. Alguns Aspectos da Situação do Idoso no Brasil. São Paulo, Imprensa Oficial do Estado, 1976 7p. UNISINOS

225 NICOLA, Ivalina Porto. Algumas Considerações sobre o Adulto Ve1ho. Momento, Rio Grand e, v. 5, p. 7-13, jan./ dez. 1992 EDU 
226 NICOLA, Pietro de. Fundamentos deG eriatriaeG erontologia. Porto Alegre, D. C. Luzzato, 1986 386p. ULBRA

227 NICOLETTO, Ugo. Ansiedade Implicada em Níveis de Ocupação em Sujeitos de Terceira Id ade. Enfoque, Bento Gonçalves, v. 15, n. 65, p. 3-21, jun. 1987. EDU

228 NOÇÕES Práticas de Geriatria. Belo Horizonte, Health, 1994. 419 p. PSICO

229 NOGUEIRA, E. E. T.; MEDINA, M. F.. A Instituição Imaginária da Universidade para a Terceira Idade (UNITI): um estudo das representações de alguns de seus membros. In: SALÃ O DE INICIAÇÃ O CIENTÍFICA, 5., 1993, Porto Alegre [Resumos]. Porto Alegre, UFRGS, 1993 P. 261. PSICO

230 NUNES, Catarina Pillar. Ocorrência de Doenças Cardíacas em Gerontes Atendidas no Hospital de Pronto Socorro Municipal de Porto Alegre. Porto Alegre, UFRGS/Curso de Pós-Graduação em Enfermagem, 1979. 46 p. ENF

231 ADULTO mais Idoso: uma abordagem fisiológica. Sprint Magazine, Rio de Janeiro, v. 11, n. 59, p. 6-8, mar./abr. 1992 ESEF

232 PAPEL da Família. Correio da Unesco, São Paulo, v. 10, n. 12 p. 21, dez. 1982 UNISINOS

233 ODORIZZI, Cármen Maria Andrade. Uma Pedagogia paraa Velhice: o desafio da construção de um trabalho com idosos no Brasil. Porto Alegre, PUCRS, 1996. 248f. PUCRS

234 OKUMA, Silene. O Idoso e a Atividade Física; fundamentos e pesquisa. Campinas, Papirus, 1998 208p. UNISINOS

235 OLIVEIRA, Elizabeth de Viegas. Alimentação e Nutrição em Geriatria. RevistaABIA SAPRO., n. 49, p. 10-19, ago. 1980. ICTA

236 OLIVEIRA, J. E. Abreu de. Aposentadoria noServiço Público: direito do funcionário, prestação do estado, legislação doutrina e jurisprudência. Rio de Janeiro, Freitas Bastos, 1970. 554p. PUCRS

237 OLIVEIRA, Rita de Cássia da Silva. Universidade Aberta paraa Terceira Idade na Universidade Estadual. Santiago de Compostela, s. n, 1998 635f ULBRA 
238 OLSZEWER, Efraim. Envelhecer com Saúde. São Paulo, Nova Linha, 1994 123p. UNISINOS

239 OS CENTENÁRIOS dos Altos Vales. Correio da Unesco, São Paulo, v. 10, n. 12 p. 18 19, dez. 1982 UNISINOS

240 OS SÁBIOS Centenários dos And es. Ciência e Cultura, São Paulo, v. 28 , n. 6, p. 704, jul. 1976. UNISINOS

241 OTTO, Edna Ruth de Castro. Exercícios Físicos para a TerceiraIdade, São Paulo, Manole, 1987. 95p. ESEF-IPA/IMEC

242 PAPALÉO NETO, Matheus. G erontologia São Paulo, Atheneu, 1996. 524p. IPA/IMEC

243 PAZ, Raul Lorda. Educação Física e Recreação para a Terceira Idade. Porto Alegre, Sagra Luzatto, 1990. 135p. ULBRA-IPA/IMEC

244 PELO PRISMA dos Jovens. Correio daUnesco, São Paulo, v. 10, n. 12 p. 22-27, dez. 1982 UNISINOS

245 PENTEADO, Cláudia. Cresce Oferta de Produtos e Serviços para a Terceira Idade. Meio Mensagem, São Paulo, v. 17, n. 637, p. 2425, jan. 1995. FBC

246 PEREIRA, Rosane Carrion Jacinto; WALDOW, Vera Regina. Identificação da Opinião dos Alunos de Enfermagem a Respeito de Campos de Estágio com Idosos. RevistaG aúcha deEnfermagem, Porto Alegre, v. 9, n. 2 p. 105-112 ENF

247 PERNISA, Hamlet. Atividades Físicas e Recreativas com Indivíduos na $3^{a}$ Idade. Sprint Magazine, Rio de Janeiro, v. 5, n. 47, p. 30-31, mar./ abr. 1990. ESEF

248 PETRY, Tiago; FOLBERG, Maria Nestrivsky, (Orient.). In: SALÃ O DE INICIAÇÃ O CIENTÍFICA, 5., 1993, Porto Alegre. [Resumos]. Porto Alegre, UFRGS, Pró-Reitoria de Pesquisa e Pós Grad uação, 1993. p. 262EDU

249 PICON, Pedro Dornelles, et al. Esquema de Tuberculostáticos em Pacientes Idosos no Tratamento Quimioterápico da Tuberculose. Rio de Janeiro, São Paulo, MEDSI, 1993 p. 559-565MED

250 PIKUNAS, Justin. Human Development: an emergment science. New York, Mcgraw-Hill, 1976. 435p. EDU 
251 PIRES, Roberto. Cidadania não tem Idade. Tempo e Presença, São Paulo, v. 14, n. 264, p. 25-27, jul./ ago. 1992 EDU

252 PORTELA, Vera Catarina Castiglia. O Trabalho Profissional doEnfermeiro. Rio de Janeiro, Universidade do Estado, 1988 114p. ENF

253 PORTO, Celmo Celeno (Org.) ExameClínico. Rio de Janeiro, Guanabara Koogan, 1996. 465p. MED

254 POZZEBON, Inês; DALCIN, Mariângela P. Exercícios xOsteoporose. Porto Alegre, UFRGS/ESEF, 1996. 31f. ESEF

255 PRETTI, Dino. A Linguagem dos Idosos; um estudo da análise da conversação. São Paulo, Contexto, 1991. 126p. UNISINOS-ULBRA

256 PRETTI, Dino. As Narrativas na Interação de Idosos. Porto Alegre, ANPOLL, 1991. p. 349-358 BSCHC

257 PREVIDÊNCIA Social Inerente à Sociedade. Ciência e Cultura, São Paulo, v. 25, n. 1, p. 33-34, jan. 1973. UNISINOS

258 PUSTAI, Erna Maria. Terceira Idade; envelhecer com dignidade. São Leopoldo, Unisinos, 1992 103p. UNISINOS

259 RABELLO, Ernesta S., et al. Enfermagem no Grupo Interdisciplinar de Gerontologia. Revista G aúcha deEnfermagem, Porto Alegre, v. 11, n. 2 p. 47-51, jul. 1990. ENF

260 RAMOS, Alexandre Trindade. Atividades Físicas: diabéticos, gestantes, $3^{\mathrm{a}}$ idade, crianças, obesos. Rio de Janeiro, Sprint, 1997. 114p. IPA/IMEC

261 RAMOS, Terezinha Auxiliad ora Gonzaga. Acid entes na Velhice: aspectos epidemiológicos. Revista Baiana deEnfermagem, Salvador, v. 2 n. 1, p. 140 141, 1986 ENF

262 RAMOS, Terezinha Auxiliadora Gonzaga. Uso de Medicamentos por Pacientes Idosos. Revista Baiana deEnfermagem, Salvador, v. 3, n. 1/2 p. 8494, jun./dez. 1987. ENF

263 RAUCHBACH, Rosemary. A Atividade Física paraa TerceiraIdade. Curitiba, Lovise, 1990. 110p. UNISINOS

264 REZENDE, Ana Lúcia Magela de. O Paciente Geriátrico Face à Cirurgia. Revista Brasileira deEnfermagem, São Paulo, v. 29, n. 1, p. 38-47, jan./ mar. 1976 ENF

265 RIKKERS, Renate. Seniors on the Move. Champaign, Human Kinetics, 1986. 240p. ESEF 
266 RIO GRANDE DO SUL. Conselho Estadual do Idoso. Os Idosos do Rio G rande do Sul: estud o multidimensional de suas condições de vida: relatório preliminar de pesquisa. Porto Alegre, O Conselho, 1996 66f. PSICO 267 RODRIGUES, Rosalina A. P.; DIOGO, Maria José D. ComoCuidar dos Idosos. Campinas, Papirus, 1996. 125p. CEDOPE-ENF

268 RODRIGUES, Rosalina Aparecida Partezani, MENDES, Maria Manuela Rião. Incontinência Urinária em Idosos: proposta para a conduta da enfermeira. RevistaLatino-Americana deEnfermagem, RibeirãoPreto, v. 2, n. 2, p. 5-20, jul. 1994. ENF

269 RODRIGUES, Rosalina Aparecida Partezani; ZAGO, Márcia Maria Fontão. Incontinência Urinária do Idoso: assistência de enfermagem. Revista da Escola de Enfermagem daUSP, São Paulo, v. 25, n. 1, p. 21-28, abr. 1991. ENF

270 RODRIGUEZ, Cristian Mercado; HEKMAN, Paulo Rogério Wasserstein. Pirexia Silenciosa no Idoso: mito ou realidade. Revista de Medicina da PUCRS, Porto Alegre, v. 4, n. Esp., p. 15, 1994. PUCRS

271 ROMERO, Elaine. Mulheres em Movimento. Vitória, Universidade Fed eral do Espírito Santo, 1997. 300p. UNISINOS

272 ROSA, Adriane Maria Gonçalv es da. Terceira Idade e ServiçoSocial: uma abordagem prática em construção. São Leopoldo, Unisinos, 1993. 53p. UNISINOS

273 ROSA, Maria Inês. "Velhice" do Trabalhador e Relações de Traba1ho. Tempo e Presença, São Paulo, v. 14, n. 264, p. 118-21, jul./ago. 1992. EDU

274 ROSA, Maria João Valente. O Desafio Social do Envelhecimento Demográfico. Análise Social, Lisboa, v. 38, n. 122, p. 679-689, 1993. UNISINOS

275 ROSA, Merval. PsicologiaEvolutiva Petrópolis, Vozes, 1983 4v. EDU 276 ROVEDA, Mari Cleusa. Terceira Idade. São Leopoldo, Unisinos, 1992 171 p. UNISINOS

277 ROZESTRATEN, Reinier J. A.. O Idoso, suas Capacidades Psíquicas e o Trabalho. Ciência e Cultura, São Paulo, v. 40, n. 7, p. 673-679, jul. 1988. EDU 
278 RUBIN, Isadore. Sexual Life After Sixty. New York, Basic Books, 1965. 274p. ULBRA

279 SÁ, Sulmara Moreira de. Fatores Psicossociais que Desencadeiam a Desnutrição na Terceira Idade. São Leopoldo, Unisinos, 1993. 40 p. UNISINOS

280 SACHS, Judith; SMITH, Dennis; FROES, Paulo (Adapt.) Menopausa: o que as mulheres devem saber. Rio de Janeiro, Ediouro, c 1994 128p. PUCRS

281 SALGADO, Marcelo Antônio. A Questão Social do Idoso no Brasil. Intercâmbio, Rio de Janeiro, v. 3, n. 7/ 9, p. 5-11, jan./ dez. 1990. EDU

282 SALGADO, Marcelo Antônio. GerontologiaSocial: teorias do envelhecimento; a aposentadoria e ética. Rio de Janeiro, CBCISS, 1993 93p. PUCRS

283 SAND, Gayle. Está Quente Aqui ou Sou Eu? um exame pessoal dos fatos, equívocos e sensações da menopausa. São Paulo, Summus, 1995. 165 p. PUCRS

284 SANTANA, Anderson. Os Benefícios da Natação para a Terceira Idade. Sprint Magazine, Rio de Janeiro, v. 11, n. 61, p. 36-39, jul./ago. 1992 ESEF

285 SANTOS, Cláudio José dos. Um Caminho de Vida. Santos, Leopoldianum, 1997. 112p. ULBRA-CEDOPE

286 SANTOS, Cláudio José dos. Universidade Aberta para a $33^{a}$ Idade: um caminho de vida. Santos, Leopoldianum, 1997. 112p. PUCRS

287 SANTOS, Geraldine Alves dos. Planejamentode Vida, Ansiedade e Tensões Psíquicas nas Pessoas da Terceira Idade. Porto Alegre, PUCRS, 1996. 135f. PUCRS

288 SANTOS, Maria de Fátima de Souza. Identidade e Aposentadoria. São Paulo, EPU, 1990. 87p. PUCRS

289 SANTOS, Marisa Noal dos. Envelhecimento; o exercício físico beneficiando este processo. São Leopoldo, Unisinos, 1993 69p. UNISINOS

290 SANTOS, Silvana Berten dos. O Lazer como Instrumento de Participação Junto a Idosos Institucionalizados; uma prática do serviço social. São Leopoldo, Unisinos, 1993، 102p. UNISINOS

291 SCHACHTER SHALOMI, Zalman; MILLER, Ronald S. Mais Velhos, Mais Sábios; uma visão nova e profunda da arte de envelhecer. Rio de Janeiro, Campus, 1996. 318p. UNISINOS 
292 SCHAIE, R. Wamer; WLLIS, Sherry L. AdultDevelopment and Aging. New York, Springer, 1980. 176p. ULBRA

293 SCHARLL, Martha. Actividad física en laterceraedad. Rio de Janeiro, Cultura Médica, 1978. 41 p. ULBRA

294 SCHARLL, Martha. Como Manter-se Ativo na Velhice. Rio de Janeiro, Cultura Médica, 1978 41 p. IPA/IMEC

295 SCHISLER FILHO, William. A Ilha dos Id osos Felizes. Tempo e Presença, São Paulo, v. 14, n. 264, p. 35-36, jul./ago. 1992. EDU

296 SCHNEIDER, J. Manual de Geriatria. São Paulo, Roca, 1985. 433p. IPA/IMEC

297 SCHONS, Silvana A. F. Terceira Idade; perfil de 46pessoas na faixa etária de 50a mais de 80anos moradores do município de Montenegro-RS. São Leopoldo, Unisinos, 1995. 92p. UNISINOS

298 SCHRAIBER, Lilia Blima; NEMES, Maria Ines Baptistella; MENDES GONÇALVES, Ricardo Bruno. Saúde do Adulto; programas e ações na unidade básica. São Paulo, UCITEC, 1996. 290p. UNISINOS

299 SEMINÁRIO DOS PROFISSIONAIS DE EDUCAÇÃ O FÍSICA QUE, ATUAMCOMATIVIDADESFÍSICAS PARA A TERCEIRA IDADE, 1., 1989, PORTO ALEGRE). [Síntese Teórico Prática]. Porto Alegre, Assessoria de Comunicação Social da Fesc/Administração Popular de Porto Alegre, 1991. 55p. ESEF

300 SERRO AZUL, Luiz Gastão. Clínicado Indivíduo Idoso. Rio de Janeiro, Guanabara Koogan, 1981. 352p. IPA/IMEC-ULBRA

301 SERVIÇO Social do Comércio. Ad ministração Regional no Estado de São Paulo (Ed). Cadernos da Terceira Idade. São Paulo, SESC, 1987. 145p. ENF

302 SERVIÇO Social do Comércio. Bibliografia Gerontologiae G eriatria: artigos de periódicos. São Paulo, SESC, 1989. 24p. ENF

303 SHEPHARD, Roy J. ; ASTRAND, Per-Olof, (Eds.) Endurance in Sport. Oxford, Blackw ell, 1992 638p. ESEF

304 SHUI, Yi. Um Assunto de Família. Correio da Unesco, São Paulo, v. 10, n. 12, p. 16-17, dez. 1987. UNISINOS

305 SILVA, Antônio Carlos Pacheco E.. Envelhecer sem Esmorecer. São Paulo, Melhoramentos, 1978. 118p. UNISINOS 
306 SILVA, José Anísio da. O Idoso no Brasil: novas propostas. Intercâmbio, Rio de Janeiro, v. 4, n. 10, p. 15-25, jan./ abr. 1991. EDU

307 SILVERSTEIN, Alvin. Conquista daMorte. São Paulo, Difel, 1981. 189 p. UNISINOS

308 SIMÕES, Antônio. Alguns Mitos Respeitantes ao Idoso. RevistaPortuguesa de Pedagogia, Coimbra, v. 24, p. 109-121, 1990. EDU

309 SIMÕES, Antônio. Aspectos do Desenvolvimento Cognitivo do Idoso. Revista Portuguesa de Pedagogia, Coimbra, v. 25, n. 2, p. 177-185, 1991. EDU

310 SIMÕES, Regina. Corporeidade e Terceira Idade: a marginalização do corpo id oso. Piracicaba, Unimep, 1998131 p. IPA/IMEC

311 SKINNER, Burrhus Frederic; VAUGHAM, M.E. Viva Bem a Velhice. São Paulo, Summus, 1985. 141 p. EDU

312 SMITH, Marília de Arruda. Aspectos Citogenéticos do Processo de Envelhecimento Celular. Ciência eCultura, São Paulo, v. 4, n. 7, n. 659-665, jul. 1988. UNISINOS

313 SODEMAN, Willian A. Instructions For Geriatric Patients. Philadelphia, W. B. Saund ers, 1995. 340p. PUCRS

314 SOVA, Ruth. Hidroginástica na Terceira Idade. São Paulo, Kianole, 1992. 207p. IPA/IMEC

315 SPERB, Dalilla Clementina. O Ano Nacional do Idoso. Revista deEstudos, Novo Hamburgo, v. 5, n. 1, p. 1823, jun. 1982 EDU

316 STEENVOORDEN, M. A. G. A.. Prise en Charge Par la Famille des Personnes Tres Agees: recueil d'intiatives. Dublin, Loughlinstown House, Shankill, 1993. 81 p. DIR

317 STORRS, Alison M. F. Enfermagem Geriátrica. Lisboa, Publicações Europa-America, 1980. 235p. UNISINOS

318 TELFORD, Charles W.; SA WREY, James M. O Indivíduo Excepcional. Rio de Janeiro, Guanabara, 1988 658p. ENF

319 TERRA, Newton Luiz; JOTZ, Jose Carlos Pereira. Câncer Coloretal no Idoso. Revista de Medicina da PUCRS, Porto Alegre, v. 1, n. 3, p. 120 126, 1989. PUCRS

320 TFAUNI, Dirce Fátima Vieira. Terceira Idade e a Felicidade Merecida. Viver Psicologia, v. O, n. 17, p. 11-13, 1986 PUCRS 
321 UM MUNDO que Envelhece: alguns dados estatísticos. Correio da Unesco, São Paulo, v. 10, n. 12 p. 20, dez. 1982 UNISINOS

322 UMPLANO Mundial de Ação. Correio da Unesco, São Paulo, v. 10, n. 12 p. 32-34, dez. 1982 UNISINOS

323 UMA CONVERSA Franca Sobre a Arte de Envelhecer. Ribeirão Preto, Sociedade Brasileira de Psiquiatria Clínica, 1991. IPA/IMEC

324 UNITED NATIONS INTERNATIONAL CONFERENCE ON AGEING POPULA TIONS IN HE CONTEXT OF URBANIZATION, 1988 Aging and Urbanization: Proceedings. New York, United Nations, 1991. 46 p. DIR

325 UNITED NATIONS. Plan de Accion Internacional de Viena sobre Envejecimiento. New York, 1983 62p. DIR

326 UNITED NATIONS. DEPARTAMENT OFECONOMIC SUBJECTAND INTERNA TIONAL SOCIAL. The World Aging Situation: strategies and policies. New York, 1985. 301 p. DIR

327 UNITED NATIONS. DEPARTAMENT OF ECONOMICSAND SOCIAL SUBJECT. The Aging: trends and policies. New York, United Nations, 1975. 103p. DIR

328 UNIVERSIDADE FEDERAL DO RIO GRANDE DO SUL. Escola de Enfermagem. Geriatria: aspectos sociais. Porto Alegre, UFRGS/Escola de Enfermagem, [1983?]. 1v. ENF

329 VANZIN, Arlete Spencer; NERY, Maria Elena da Silva (Org.) Estudo deIndicadores Sociais em G erontes Residentes em Casas G eriátricas em 1990 Porto Alegre, ABEN, 1990. 33f. ENF

330 VARGAS, Heber Soares. Psicologia do Envelhecimento. São Paulo, Byk-Procienx, 1983. 150p. UNISINOS-IPA/IMEC

331 VENCENDO a Velhice. São Paulo, Zumbi, 1958. 144p. UNISINOS

332 VITAL, José. O INPS e a Assistência aos Idosos. São Paulo, Instituto Nacional de Previdência Social. 1976. 8p. UNISINOS

333 VITOLA, Janice de Oliveira Castilhos. Terceira Idade: tendência atualizante e sentido de vida. Porto Alegre, PUCRS, 1997. 120f. PUCRS

334 WAGNER, Elvira Mello. Amor, Sexoe Morte noEntardecer da Vida. São Paulo, Caiçara, 1989. 153p. IPA/IMEC 
335 WALDOW, Vera Regina. A Enfermagem como Agente de Mudança na Percepção do Envelhecimento. Revista do Hospital de Clínicas de Porto Alegre. Porto Alegre, v. 3, n. 1, p. 117-120, jun. 1983 ENF

336 WALDOW, Vera Regina. A prendizagem na Velhice. RevistaG aúcha de Enfermagem, Porto Alegre, v. 3, n. 1, p. 71-80, 1981. ENF

337 WALDOW, Vera Regina. Aprendizagem na Velhice: aplicação das teorias de Rogers, Maslow e Brunner. Revista Gaúcha de Enfermagem, Porto Alegre, v. 8, n. 2 p. 244260, 1987. ENF

338 WALDOW, Vera Regina. Determinação do Nível de Dependência em Enfermagem junto a Idosos Institucionalizados de Porto Alegre/ RS. Revista Gaúcha de Enfermagem, Porto Alegre, v. 8, n. 1, p. 46-73, 1987. ENF

339 WALDOW, Vera Regina. Determinação do Nível de Dependência em Enfermagem Junto a Idosos Institucionalizados de Porto Alegre/RS. Rio de Janeiro, ABEN, 1986 p. 68ENF

340 WALDOW, Vera Regina. O Papel da Enfermagem na Velhice em Face das Modificações Fisiológicas e Fisiopatológicas. Revista Paulista de Enfermagem, São Paulo, v. 4, n. 4, p. 127-131, 1984 ENF

341 WALDOW, Vera Regina. Opinião de Idosos Acerca da Velhice. A Terceira Idade, São Paulo, v. 5, n. 8, p. 25-38, jun. 1994 ENF

342 WALDOW, Vera Regina. Problemas Sentidos porIdosos deDiferentes Classes Sociais de PortoAlegre/RS eServiços Sociais parao A tendimentoa suas Necessidades Básicas. Porto Alegre, UFRGS, 1985. 214p. ENF-EDU

343 WALDOW, Vera Regina; FRICHEMBRUDER, Renate. A Velhice: um ensaio experimental. RevistaGaúchadeEnfermagem, Porto Alegre, v. 6, n. 2. p. 275-292, 1985. ENF

344 WALDOW, Vera Regina. Determinação do Nível de Dependência em Enfermagem Junto a Idosos Institucionalizados em Porto Alegre RS. Porto Alegre, UFRGS/ Escola de Enfermagem, 1986 38p. ENF

345 WALSHE, Thomas M. Manual de problemas clínicos en medicina geriatrica: com Bibliografia Comentada. México, Interamericana, 1986 413 p. ENF 
346 WALTER, Silvia E.. Terceira Idade: perspectivas de valorização pessoal. São Leopoldo, Unisinos, 1993. 63p. UNISINOS

347 WEINECK, Jurgen. Biologia do Esporte, São Paulo, Manole, 1991. 599p. ESEF

348 WETTREICH, Wald emar. Medicina Física Reabilitação em G eriatria. [s. 1] Merca Brasil [ 19- -] ULBRA

349 WHITCOMB, Phillip W.. A Magnífica Legião dos Velhos. Correioda Unesco, São Paulo, v. 10, n. 12, p. 47, dez. 1982 UNISINOS

350 WILKE, Kurt. Palestra Curitiba, All Sport, 1991. ca. 53p. ESEF

351 WOLTERECK, Heinz. Vida Nova para Velhos. São Paulo, Ibrasa, 1959. 265p. UNISINOS

352 WOOD, Diana. Tempo de Plenitude. Sprint Magazine, Rio de Janeiro, v. 11, n. 60, p. 25-29, maio/jun. 1992. ESEF

353 WORLD HEALTH ORGANIZATION. The Control of Drugs for the Elderly. Copenhagen, WHO, 1981. 36p. FAR

354 YAZAKI,: Lúcia Mayumi. Perspectivas Atuais do Papel da Família Frente ao Envelhecimento Populacional: um estudo de caso. RevistaBrasileira de Estudos de População, São Paulo, v. 8, n. 42 p. 137-141, jan./dez. 1991. UNISINOS

355 YUM, Jon Suk. NãoHá A posentadoria na Vida: como chegar aos 120 anos. São Paulo, Convite, 1987. 272p. IPA/IMEC

356 ZANELLI, José Carlos; SILVA, Narbal. ProgramadePreparação para Aposentadoria. Florianópolis, Insular, 1996 112p. PUCRS. 